\title{
Warum eine neue Zeitschrift zu Religion?
}

\author{
Gert Pickel · Annette Schnabel
}

CC Springer Fachmedien Wiesbaden 2017

Warum eine neue Zeitschrift zu Religion? Das ist die Eingangsfrage, die man sich angesichts des vorliegenden Heftes stellen kann. Gibt es nicht genügend Zeitschriften, die sich national oder international mit Religion auseinandersetzen - und bedarf es da noch einer? Wir, die Herausgeber als auch die Mitglieder der Redaktion, meinen: ja.

So dürfte kaum zu bestreiten sein, dass das Thema Religion in den letzten Jahrzehnten einen massiven Aufschwung erfahren hat. Manch einer würde vielleicht sogar sagen, dass es zu einer Wiederkehr des Religiösen gekommen sei. Dies ist dahingehend bemerkenswert, lag davor doch eine Phase, in der nicht nur westeuropäische Gesellschaften wie selbstverständlich mit dem Absterben der Religion und globale Modernisierungsprozesse mit zunehmender Säkularisierung verbunden schienen. Sozialwissenschaftliche - und auch andere - Gesellschaftsanalysen assoziierten, spätestens seit Max Webers Schriften, Moderne mit Rationalisierung und diese mit dem Diktum der „Entzauberung der Welt“, während gleichzeitig Säkularisierungstheorien als Modernisierungstheorien verfasst wurden. Auch in den nationalstaatlichen Selbstbeschreibungen moderner Staatengebilde spielt die Trennung von Staat und Religion sowie die verfassungsmäßige Anerkennung der Menschenrechte und die darin verankerte Religionsfreiheit eine zentrale Rolle bei der Legitimation als moderner, berechenbarer Akteur innerhalb der internationalen Staatengemeinschaft. Da ist es nicht überraschend, dass gerade Debatten über das Verhältnis zwischen Kirche

G. Pickel ( $\bowtie)$

Universität Leipzig, Leipzig, Deutschland

E-Mail: pickel@rz.uni-leipzig.de

\section{A. Schnabel}

Universität Düsseldorf, Düsseldorf, Deutschland

E-Mail: annette.schnabel@uni-duesseldorf.de 
und Staat eine lange Phase der Dominanz in der Diskussion über Säkularisierung eingenommen haben - und an verschiedenen Stellen auch heute noch einnehmen.

Spätestens jedoch mit dem Jahrtausendwechsel wurde nicht nur die immer noch bestehende Existenz und kulturelle Verankerung der Religionen in der westlichen Welt erkennbar, sondern Religion und Religiosität werden weltweit zunehmend als wichtige Faktoren gesellschaftlicher und politischer Konflikte, Auseinandersetzungen und Diskurse sichtbar. Gerade Konflikte, in denen Religion und religiöse Zugehörigkeit thematisiert werden, scheinen aktuell einer sich beschleunigenden globalen, regionalen und lokalen Dynamik zu unterliegen. Auch das Verhältnis der Religionen zum Staat und religionsbezogene Kontroversen, Konfrontationen, Konkurrenzkämpfe und Auseinandersetzungen fordern insbesondere vor dem Hintergrund von Prozessen massiver Veränderungen in der religiösen Zusammensetzung von Gesellschaften zu einem neuen Klärungsbedarf dieser Wechselbeziehungen heraus. Speziell Pluralisierung - die beispielsweise westeuropäische Länder aktuell durch verstärkte Migration erleben - bringt derzeit ähnlich schwerwiegende soziale Umstrukturierungen und Umbrüche mit sich, wie die religiöse Homogenisierung durch Mission, Segregation oder Vertreibung.

Diese gesellschaftlichen Veränderungen der Sphäre des Religiösen und die damit verbundenen neuen und erweiterten sozial relevanten Fragestellungen haben in den letzten Jahren zu einer zunehmenden, gerade auch sozialwissenschaftlichen Beschäftigung speziell jüngerer Wissenschaftler und Wissenschaftlerinnen mit Theorien, Konzepten und empirischen Entwicklungen im Sektor Religion, Gesellschaft und Politik geführt. Dazu zählen eben nicht nur die klassischen Fragen nach der Häufigkeit des Kirchgangs oder der Verteilung verschiedener Religionsgemeinschaften in Gesellschaften, es gelangen vielmehr Fragen in den Fokus, die den Blick auf Religion als das Soziale und Politische in vielfältigster Weise beeinflussend und durch unterschiedlichste gesellschaftliche Kontexte Beeinflusstes lenken. Religion hat sich dabei auch jenseits der rein soziologischen Perspektive als ein sowohl mehr-dimensionales Konzept, das individuelle Praxen, Einstellungen, Emotionen und Lebensweisen umfasst, als auch auf verschiedenen Ebenen des Sozialen etablieren können. Themen, die sich mit dem Verhältnis von Religion und Gesellschaft oder Religion und Politik auseinandersetzen, konnten sich mehr und mehr in den Fokus gleichermaßen der Kulturwissenschaften, der Soziologie, der Politikwissenschaft und den Religionswissenschaften schieben. Religion ist folglich ein genuin die Fachgrenzen überschreitendes und schon in seinem Kern interdisziplinär angelegtes Feld geworden - wenn es dies nicht schon immer gewesen ist. Speziell die vielfältigen und dynamischen Wechselbeziehungen zwischen Religion und Gesellschaft, unter denen das Verhältnis zu Politik eine zentrale Bedeutung besitzt, stoßen in der Wissenschaft, wie aber mittlerweile auch (wieder) in öffentlichen Diskussionen auf ein nachhaltiges und wachsendes Interesse.

Vor dem Hintergrund dieses zunehmenden sowohl politischen und gesellschaftlichen als auch wissenschaftlichen Interesses an einer (wieder) zunehmenden weltweiten und höchst dynamischen Verschränkung von Religion, Gesellschaft und Politik scheint es sinnvoll, eine Zeitschrift zu etablieren, welche Beiträgen zu den vielfältigen Verhältnissen von Religion eine Plattform zur Veröffentlichung und Diskussion bietet. Damit wird aus unserer Sicht eine Lücke in der deutschen Zeitschriftenland- 
schaft geschlossen. Bisher wurde insbesondere entweder rein theoretischen oder allein empirischen Arbeiten die Möglichkeit zu einer sichtbaren Veröffentlichung geboten. Eine eigenständige, auf das Themenfeld der Religion zugeschnittene Zeitschrift, die sowohl theoretische als auch empirische Beiträge aus unterschiedlichen geistes- und sozialwissenschaftlichen Fachrichtungen systematisch vereint, fehlt aber bislang. Nun kann man argumentieren, dass der internationale, vornehmlich englisch-sprachige Markt vergleichbare Zeitschriften bietet. Trotzdem scheint uns, quasi ein wenig gegen den Trend in den Sozialwissenschaften, die Etablierung einer deutsch-sprachigen Zeitschrift angezeigt. Zum einen deshalb, weil die englischsprachigen Zeitschriften doch überwiegend den amerikanischen Markt bedienen und damit für die Besonderheiten der europäischen (und deutschen) religiösen Landschaft und ihrer in die europäische Sozialwissenschaft eingebundene Diskussion nur begrenzt zugänglich, anschlussfähig und an dieser auch nur wenig interessiert sind. Zum anderen ist der deutsch-sprachige sozialwissenschaftliche Community groß genug, so dass das Fehlen einer auf Religion und Religiosität fokussierten Zeitschrift tatsächlich eine spürbare Lücke im Sinne einer die verschiedenen mit Religion arbeitenden Fachbereiche verbindenden Austauschplattform markiert. Damit ist man faktisch bereits bei dem Mission Statement der Zeitschrift für Religion, Gesellschaft und Politik.

\section{Mission Statement}

Die Zeitschrift für Religion, Gesellschaft und Politik fokussiert auf die Verschränkung von Religion und Religiosität mit gesellschaftlichen und politischen Fragestellungen, Konflikten und Konstellationen. Sie ist Plattform für eine interdisziplinäre wissenschaftliche Befassung mit diesem Themenfeld: Beiträge sowohl aus den Sozialwissenschaften (Soziologie, Kultur-, Politik-, Medien- und Kommunikationswissenschaften), als auch aus den Geistes-, Human- und Rechtswissenschaften (Theologie, Psychologie, Rechtswissenschaften) sind willkommen. Es werden sowohl quantitative als auch qualitative Beiträge und Beiträge aus allen theoretischen Perspektiven akzeptiert, solange ihr Fokus ein sozialwissenschaftlicher ist. Die Zeitschrift adressiert primär eine sozialwissenschaftlich interessierte Leserschaft. Sie schließt eine Lücke im deutschsprachigen Zeitschriftenmarkt, der bisher noch kein auf Religion, Religiosität und deren sozialen und politischen Eingebundenheiten spezialisiertes Publikationsorgan hat. Die Zeitschrift antwortet explizit auf ein an aktuellen sozialen und politischen Entwicklungen orientiertes gesteigertes sozialwissenschaftliches Interesse an Religionen, ihren Kontexten, Folgen und wissenschaftlichen und alltagspraktischen Interpretationen.

\section{Themen und Themenschwerpunkte}

Die ZRGP begrüßt explizit Beiträge, die Religion nicht länger als ein eindimensionales individuelles Glaubensbekenntnis verstehen, sondern vielmehr als ein mehrdimensionales Konzept, das sowohl religiöse Überzeugungen, Weltanschauungen und 
Praxen, als auch religiöse Gruppen, Organisationen und Berufsfelder, Machtbeziehungen und Normsysteme von gesellschaftlicher Reichweite sowie Soziallehren und andere Wissensbestände umfasst. Damit schieben sich auch Fragen nach den gesellschaftlichen und den politischen Kontextualisierungen, Folgen und danach, wie sich Religion in einer zunehmend globalisierenden Welt wissenschaftlich fassen lassen kann, in den Untersuchungsfokus. Bestandsaufnahmen ebenso wie Entwicklungen, Dynamiken und Prozesse der vielfältigen Verschränkung von Religion(en) und Gesellschaft(en) und Politik sollen in den Beiträgen adressiert und damit einer breiten wissenschaftlichen Diskussion zugänglich gemacht werden können.

\section{Bandbreite der Beiträge}

Für die ZRGP sollen keine Einschränkungen hinsichtlich der theoretischen und methodischen Ausrichtung der Beiträge gelten. Es sind explizit sowohl quantitative als auch qualitative Forschungsbeiträge aus allen sozialwissenschaftlich-theoretischen Perspektiven erwünscht. Ziel ist es, speziell deutschsprachigen aber auch anderen europäischen Wissenschaftlern und Wissenschaftlerinnen die Möglichkeit für Veröffentlichungen an der Schnittstelle von Religion, Gesellschaft und Politik zu geben. Voraussetzung für eine Veröffentlichung ist jedoch, dass die Beträge einen explizit sozialwissenschaftlichen Fokus aufweisen.

Vom Selbstverständnis her versteht sich die Zeitschrift für Religion, Gesellschaft und Politik als interdisziplinär ausgerichtete Zeitschrift mit sozialwissenschaftlichem Schwerpunkt ihrer Beiträge. Dies umfasst auch Arbeiten mit sozialwissenschaftlichem Anspruch aus den Geisteswissenschaften, speziell der Theologie, der Psychologie und den Rechtswissenschaften. Als Leserkreis angesprochen wird entsprechend ein breites Spektrum an Wissenschaftlerinnen und Wissenschaftlern, aber auch Interessierte aus dem kirchlichen und privaten Sektor mit Interesse an sozialwissenschaftlichen Fragen zu Bedingungen und Wirkungen von Religion(en). Schwerpunktmäßig zielt die ZRGP auf westliche Gesellschaften (Europa, Nordamerika), was Beiträge über andere Regionen der Welt und Beiträge zu inter- und transnationalen Phänomenen und Entwicklungen nicht ausschließt. Im Zentrum soll die Veröffentlichung von research-based-articles in deutscher wie auch in englischer Sprache stehen.

\section{Struktur der Zeitschrift}

Die ZRGP arbeitet auf dem internationalen Standard eines strikten double blind peerreview Begutachtungsprozess. Damit wird der derzeitigen Nachfragestruktur gerade jüngerer Wissenschaftler und Wissenschaftlerinnen im Bereich der Sozialwissenschaften entsprochen. Dieser wird über ein Editorial-management-System abgebildet. Beiträge erscheinen online-first und als folgende, editierte Papierversion. Eine Einbindung in die deutsche Scientific Community wird durch die Anbindung an die Sektion Religionssoziologie der Deutschen Gesellschaft für Soziologie (DGS) sowie den Arbeitskreis Politik und Religion in der Deutschen Vereinigung für Politikwis- 
senschaft erreicht. Bereits hier danken wir den vielen Kolleginnen und Kollegen, die sich bereit erklärt haben an der Zeitschrift mitzuarbeiten. Wir haben uns sehr über das große Interesse gefreut.

\section{Erstes Heft}

Als Startpunkt sollte man sich immer einen Rückblick vornehmen. Entsprechend soll dieses erste Heft, was Ihnen jetzt vorliegt, quasi eine Art State of the Art in dem von uns anvisierten Themenfeld darstellen. Entsprechen haben wir Beiträge gebündelt, welche die Situation der Religionen in Deutschland, auch unter Berücksichtigung von Muslimen und dem Islam (Rauf Ceylan), zusammenfasst. Dafür wichtig ist es sich gewahr zu machen, was Religion eigentlich ist. Dies setzt eine Diskussion der Definition von Religion zwingend voraus (Detlef Pollack). Doch auch aktuelle Perspektiven auf Religion halten wir für die erste Nummer dieser Zeitschrift für angebracht: Neben einer ideengeschichtlichen angeleiteten Untersuchung des Verhältnisses von Religion und Politik mit dessen zusammenfassender Einordnung (Oliver Hidalgo) sind dies aktuelle empirische Entwicklungen der Religiosität in Deutschalnd und Europa (Gert Pickel), die unterschiedliche Konzeptionalisierung und Behandlung von Geschlecht in unterschiedlichen religiösen Kulturen (Heidemarie Winkel). Vor dem Hintergrund der aktuellen politischen Entwicklungen erscheint uns auch der Blick auf die Entwicklungen in den USA (Corwin Smidt) informativ. Die Konfrontation eines religionswissenschaftlichen Klassikers mit dem aktuellen Phänomen des Salafismus (Melanie Reddig über Peter L. Berger) könnte zudem helfen, das derzeitige Feld der Diskussionen über Religion, Gesellschaft und Politik zu kartographieren. Wir hoffen, Sie sehen dies ähnlich.

Ansonsten bleibt uns an dieser Stelle nur Sie dazu einzuladen, uns Ihre Beiträge zu schicken. Eine Zeitschrift lebt von den Einreichungen, allerdings auch von Ihrer Bereitschaft diese besser zu machen. So seien Sie gewiss, dass wir uns immer wieder einmal bei Ihnen mit der Frage nach Begutachtung melden werden. Es wäre schön, wenn Sie uns im Sinne des wissenschaftlichen Erkenntnisfortschritts hier weiterhelfen und Ihre Kollegen und Kolleginnen mit produktiven Anregungen unterstützen würden. Wir freuen uns über viele Einreichungen.

Gert Pickel und Annette Schnabel 